Article

\title{
A Further Contribution to the Study of Sagittamide A: Synthesis of a Pivotal Intermediate Belonging to a Rare L-Series
}

\author{
Anne Humbert, Karen Plé, Dominique Harakat, Agathe Martinez and Arnaud Haudrechy * \\ Institut de Chimie Moléculaire de Reims, UMR CNRS 7312, Université de Reims, BP 1039, \\ F-51687 REIMS Cedex, France; E-Mails: anne.bak@hotmail.fr (A.H.); karen.ple@univ-reims.fr (K.P.); \\ dominique.harakat@univ-reims.fr (D.H.); agathe.martinez@univ-reims.fr (A.M.) \\ * Author to whom correspondence should be addressed; E-Mail: arnaud.haudrechy@univ-reims.fr; \\ Tel.: +33-2691-3236; Fax: +33-2691-3166.
}

Received: 3 May 2012; in revised form: 13 June 2012 / Accepted: 18 June 2012 /

Published: 25 June 2012

\begin{abstract}
A key saggitamide intermediate corresponding to a rare sugar framework has been obtained. This approach should help to establish the overall configuration of more complex structures of the sagittamide family.
\end{abstract}

Keywords: sagittamide; L-altrose; L-talose; epoxide opening; asymmetric dihydroxylation

\section{Introduction}

Sagittamides A-F (1-6, Figure 1) are unprecedented marine natural products isolated from an unidentified tropical didemnid tunicate collected in Micronesia and described by Molinski [1,2]. These compounds are characterized by an $\alpha, \omega$-dicarboxylic acid (C-26 or C-28) that is acylated at each terminus by a different amino acid (L-ornithine or L-lysine and L-valine), and contain a stereocluster of contiguous polyacetoxy 5,6,7,8,9,10-hexaols. After a first prediction of the stereochemistry of Sagittamide A 1 using a detailed ${ }^{1} \mathrm{H}-\mathrm{NMR}$ analysis of the ${ }^{3} J_{\mathrm{H}, \mathrm{H}}$ profiles [3], a second publication gave another stereochemical attribution, discussing a similar approach but reaching different conclusions (with 2D heteronuclear NMR experiments) [4], and finally a third publication extended the uncertainty, describing three other structures (only 1a being compatible with Residual Dipolar Coupling Enhanced NMR) [5]. 
Figure 1. Structures proposed for Sagittamides A-F 1-6.

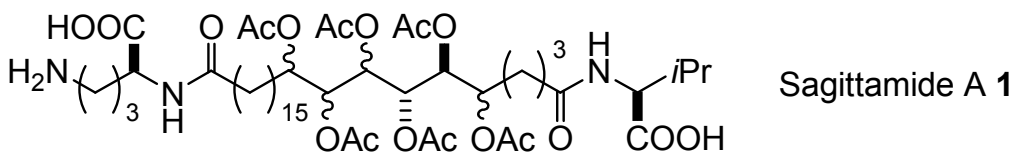

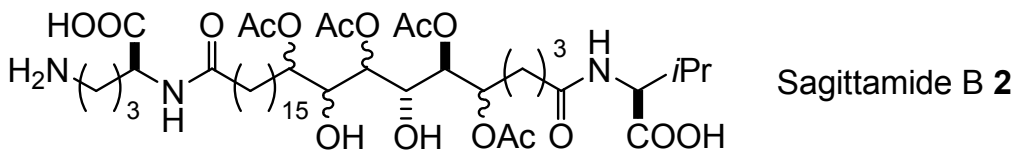

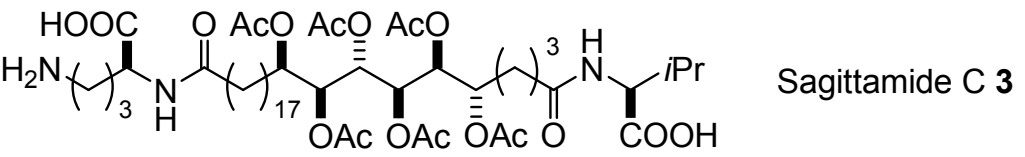

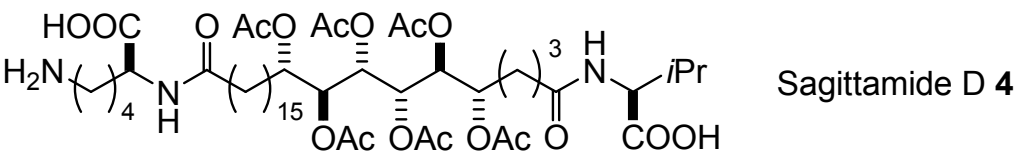

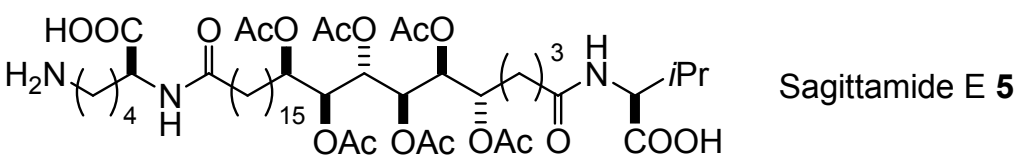

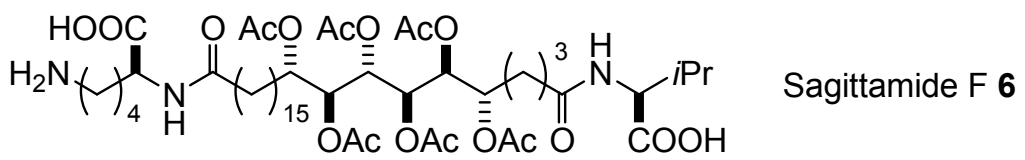

When regarding the exact configuration of Sagittamides A and B (1 and 2, Figure 1), two points have been firmly established: (a) the configuration is $S$ in positions 6 and 7; (b) There is a clear $s y n$ relationship between positions 9 and 10 [5]. Thus, the five possible structures proposed for Sagittamide A 1 are described in Figure 2.

Figure 2. Structures proposed for Sagittamide A 1.

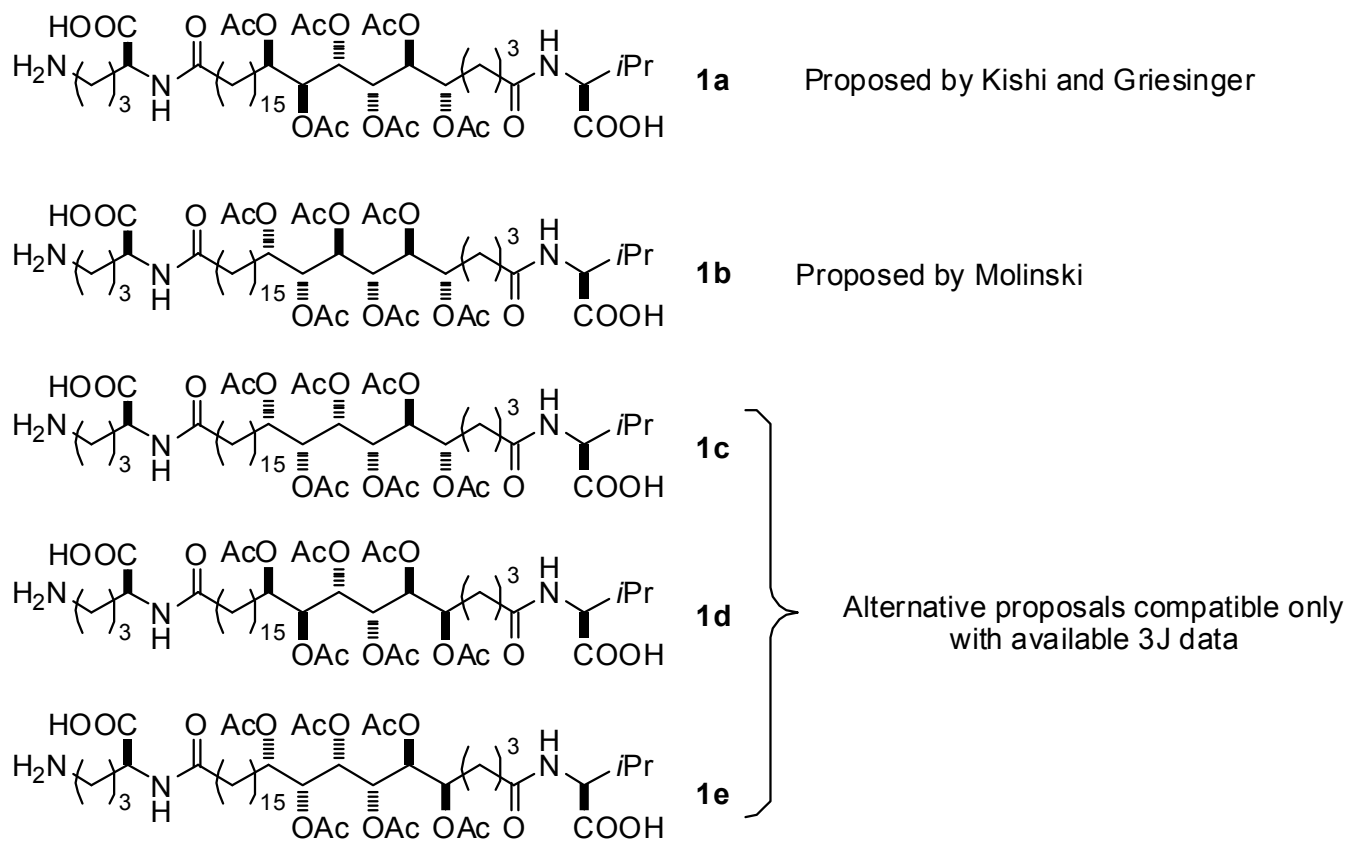


Using the Quiral program developed in our laboratory [6,7], which analyzes the 3D structure of a target organic molecule to find which sugar(s) can be used as a starting material for its synthesis, a careful study of the configurations for the central stereocluster of sagittamide A $\mathbf{1}$ (and related 2) was performed. For each possible structure (compounds 1a-e, 3-6), three groups of six consecutive carbons were analyzed, and their similarity to a known sugar identified in both directions (left to right and right to left, see Figure 3). The large majority of the proposed structures involve L-sugars, as seen in the summary table.

Figure 3. Use of the Quiral program for the study of carbohydrate backbones in the central stereoclusters of Sagittamides A-F.
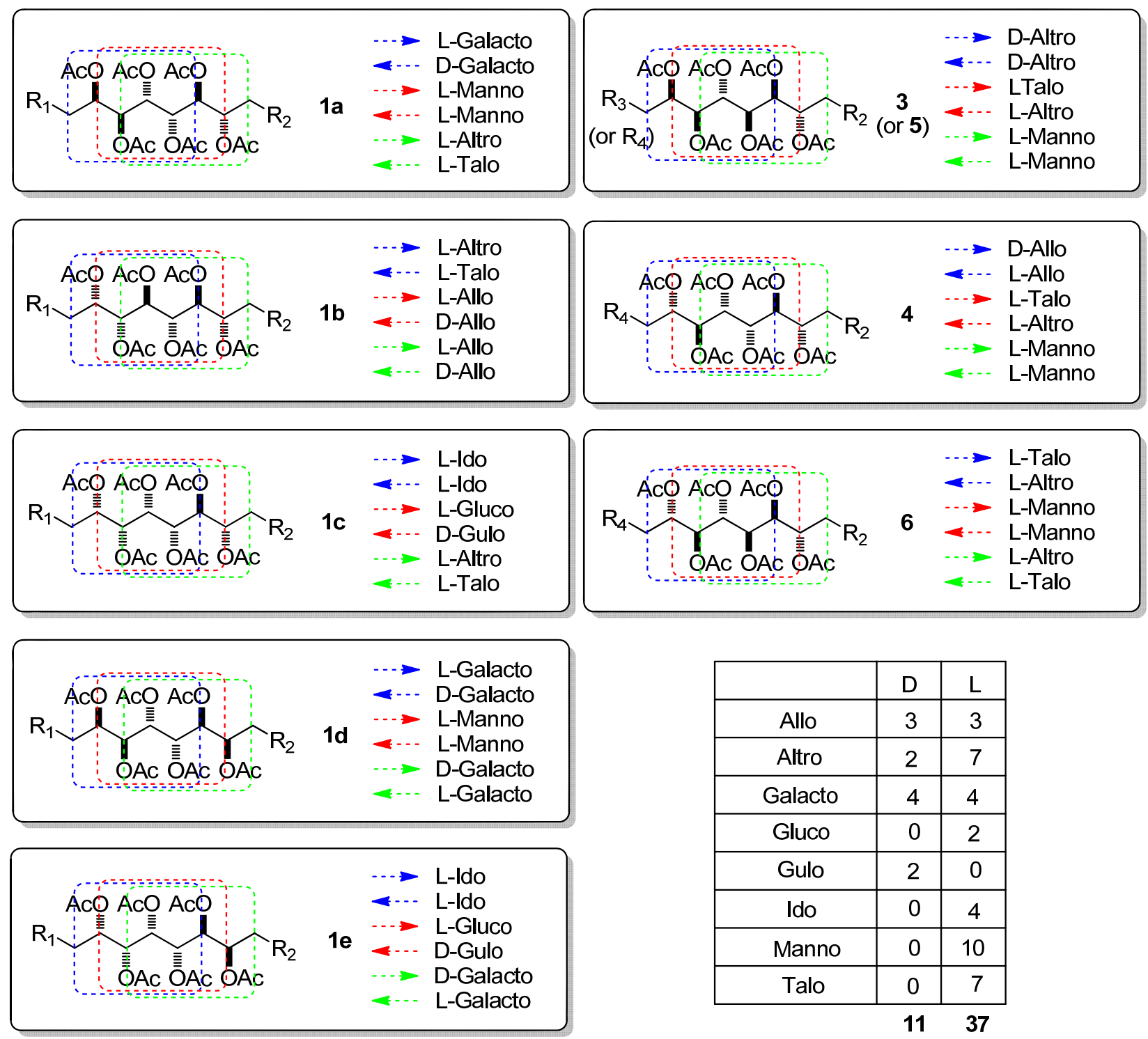

\begin{tabular}{|c|c|c|}
\hline & $\mathrm{D}$ & $\mathrm{L}$ \\
\hline Allo & 3 & 3 \\
\hline Altro & 2 & 7 \\
\hline Galacto & 4 & 4 \\
\hline Gluco & 0 & 2 \\
\hline Gulo & 2 & 0 \\
\hline Ido & 0 & 4 \\
\hline Manno & 0 & 10 \\
\hline Talo & 0 & 7 \\
\hline
\end{tabular}

Of the nine possible carbohydrate structures shown in Scheme 3, the rare sugar couple L-altrose/ L-talose can be seen in seven cases $(\mathbf{1 a - c}, \mathbf{3 - 6})$. It thus appeared feasible to start from this backbone in order to access the greatest number of structures, with any missing targets being obtained by a Mitsunobu approach. Our research was focused on finding a controlled access to the diversely protected structure 7 (Figure 4). 
Figure 4. Targeted structure 7.

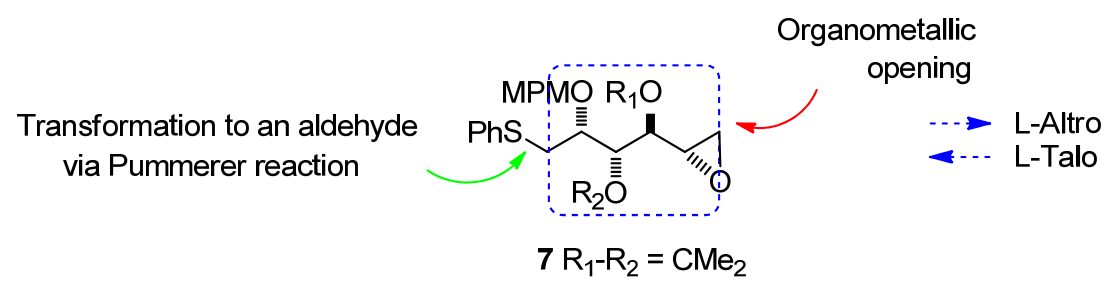

On the left-hand side of the target structure 7, the thiophenyl group is an aldehyde surrogate through a Pummerer transformation, and on the right-hand side, the epoxide could be selectively opened by diverse organometallic species.

\section{Results and Discussion}

The protected derivative 12 was easily obtained after several straightforward reactions of the known D-ribose derivative 8 (Scheme 1) [8]. After a Wittig reaction on the lactol moiety in the presence of DMSO, the previously described open carbohydrate 9 [9] was obtained in $81 \%$ yield. Noteworthy, the use of potassium tert-butoxide in THF, as described in the literature [9], resulted in a substantial loss of the TBDPS group. After several unsuccessful Mitsunobu attempts under various conditions (DEAD or DIAD as activators, toluene or THF as a solvent, $p \mathrm{NO}_{2} \mathrm{PhCOOH}$ or $\mathrm{ClCH}_{2} \mathrm{COOH}$ as nucleophilic moieties) [10-12], and inspired by some of our preliminary results [13-15], the necessary inversion of configuration occurred very cleanly under mesylate activation, followed by an in situ formation of the known epoxide 10 [16]. The direct opening of this epoxide with sodium phenylsulfide offered an efficient access to the expected derivative 11 [17]. Finally, in order to install the left-hand side of our target, the free hydroxy function was protected with a MPM group to form 12. This procedure was clean and reproducible on a multigram scale.

Scheme 1. Synthesis of the alkene derivative $\mathbf{1 2 .}$

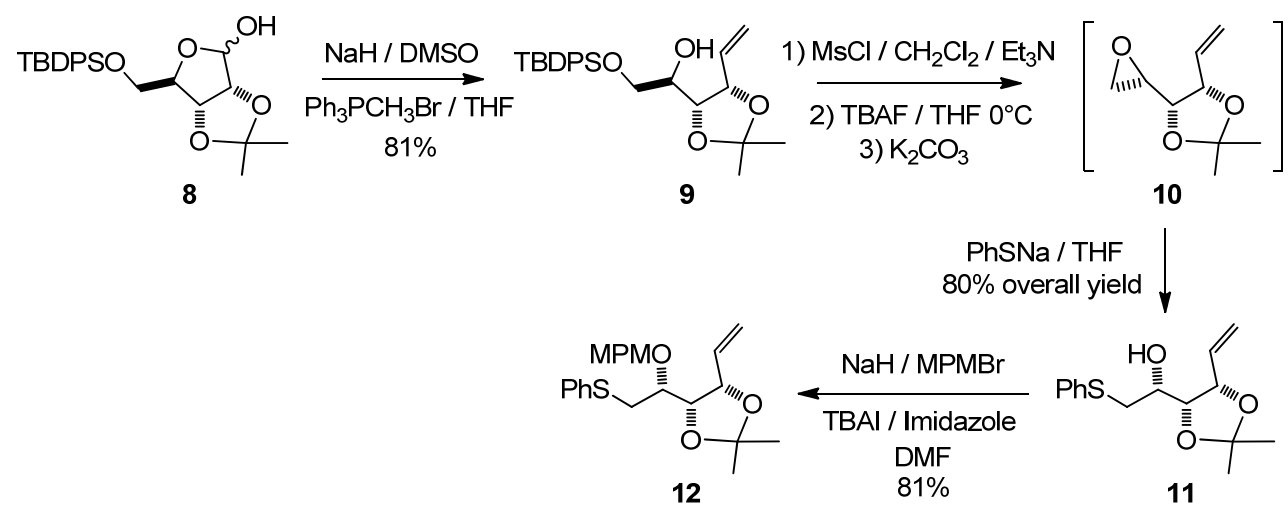

Not surprisingly, the controlled oxidation of alkene 12 with one equivalent of $\mathrm{MCPBA}$ in $\mathrm{CH}_{2} \mathrm{Cl}_{2}$ very cleanly gave the corresponding sulfoxide in $84 \%$ yield. An obvious way to finalize the synthesis involved the selective osmylation of the derivative 12. The use of osmium tetroxide $\left(\mathrm{OsO}_{4}\right)$ seemed unsuitable because of the concurrent reactivity of the sulfur. However, precedents in the literature were found in which an AD-Mix type approach was effective for this transformation [18-22]. Thus, after a first attempt in a $t \mathrm{BuOH} /$ water mixture at $0{ }^{\circ} \mathrm{C}$ that gave no reaction, the use of $\mathrm{AD}-\mathrm{Mix} \beta$ in 
acetone/water gave the $S$ diastereoisomer as the major one, in $65 \%$ overall yield (d.r. $=80: 20)$ with $11 \%$ of recovered starting material (Scheme 2). Interestingly, the use of AD-Mix $\alpha$ gave the same major diastereoisomer, with a better diastereoselectivity $(7.3: 1)$, however in lower yield (54\% overall yield, with $20 \%$ of recovered starting material). This observation was quite surprising, even if a similar case has previously been reported in the literature [23].

Scheme 2. Selective osmylation in the presence of a sulfur moiety and formation of MPA esters.

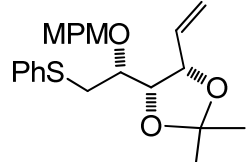

12

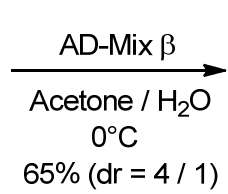

$65 \%(\mathrm{dr}=4 / 1)$

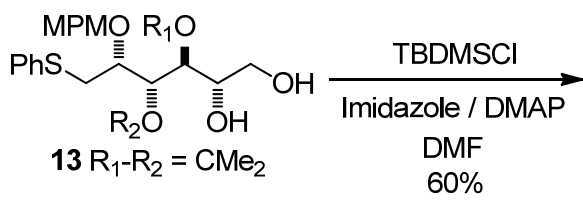

PhS $\underbrace{\mathrm{R}}_{\mathrm{R}_{2} \mathrm{I \overline { \overline {I } }}} \underbrace{\mathrm{R}_{1} \mathrm{O}}_{\text {OTH }}$ OTBDMS

$14 \mathrm{R}_{1}-\mathrm{R}_{2}=\mathrm{CMe}_{2}$
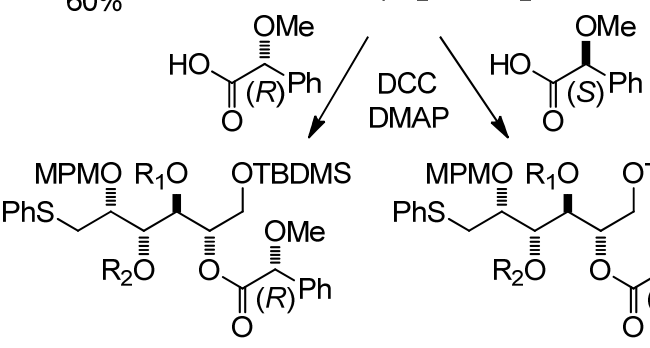

$15 \mathrm{R}_{1}-\mathrm{R}_{2}=\mathrm{CMe}_{2}$

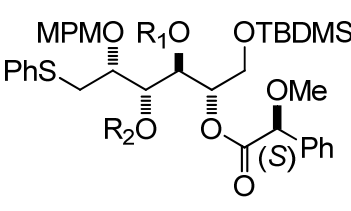

$16 \mathrm{R}_{1}-\mathrm{R}_{2}=\mathrm{CMe}_{2}$

After protection of the primary hydroxyl group with a TBDMS ether, the secondary alcohol 14 was esterified with $(R)$ and $(S)$-MPA moieties, in order to prove the induced stereoselectivity (Figure 5) [24].

Figure 5. Comparison of selected ${ }^{1} \mathrm{H}-\mathrm{NMR}$ spectra of MPA ester derivatives of $\mathbf{1 4}$.

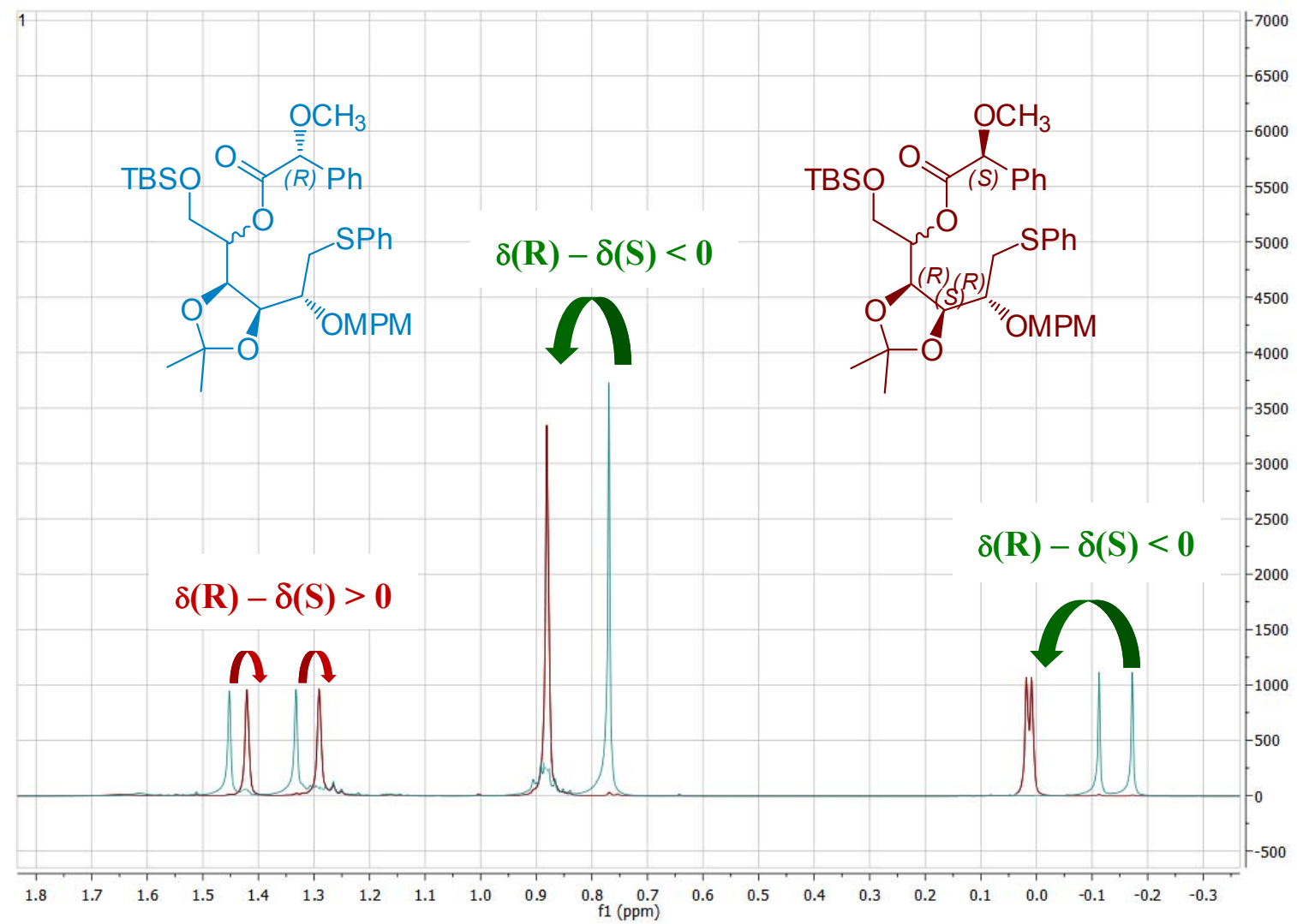


With this method, comparison of the chemical shifts of the signals due to protons in $\mathrm{L}_{1}$ and $\mathrm{L}_{2}$ in both the $R$ and the $S$ derivatives and calculation of the corresponding differences expressed as $\Delta \delta R / S$, helps to assign the $S$ configuration (Scheme 3). Finally, the desired epoxide 7 was obtained after selective intermediate tosylate activation, followed by a basic cyclization (Scheme 4). In our future synthesis of sagittamide-type structures, this epoxide could be directly opened as in one of our previously described transformations (10 to $\mathbf{1 1})$.

Scheme 3. General scheme for the assignment of $R$ or $S$ configuration.

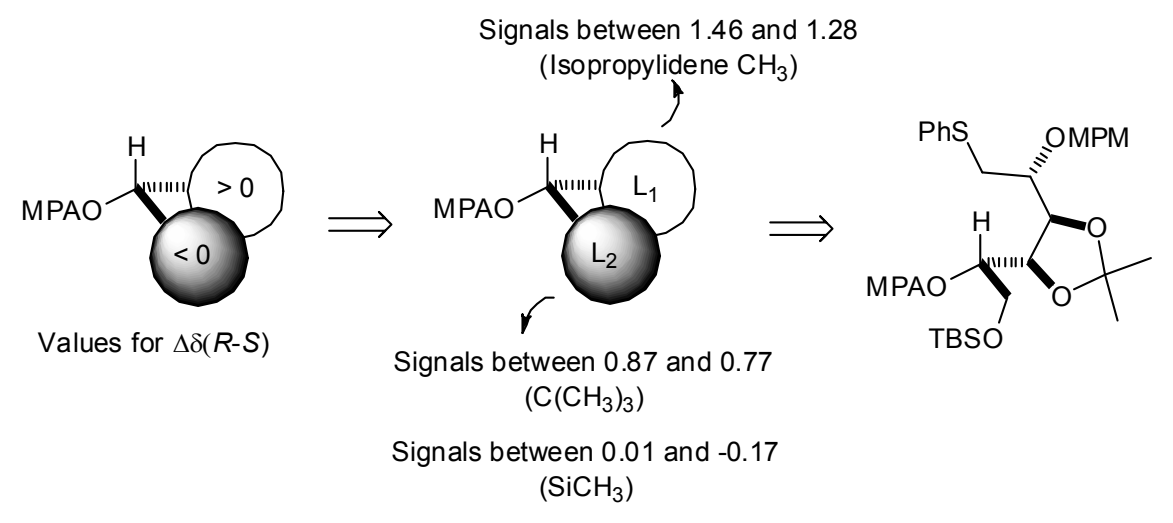

Scheme 4. Formation of target compound 7.

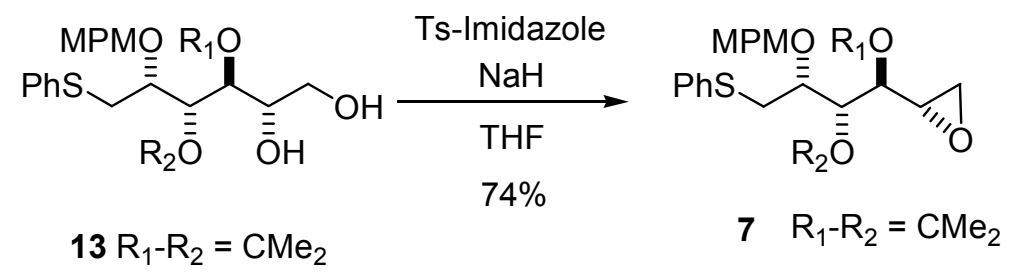

\section{Experimental}

\subsection{General}

All reactions were performed under argon with magnetic stirring unless otherwise specified. Dry solvents were used in all experiments. Thin layer chromatography was performed on E. Merck pre-coated $60 \mathrm{~F}_{254}$ plates and compounds were observed by UV or by charring the plates with an acidic anisaldehyde system. Flash chromatography was performed on an Armen SpotFlash with silica gel 60 (particle size $15-40 \mu \mathrm{m}$ ). Optical rotations were measured on a Perkin-Elmer 341 polarimeter at $20{ }^{\circ} \mathrm{C}$. Electrospray ionization mass spectrometry experiments (MS and HRMS) were obtained on a hybrid tandem quadrupole/time-of-flight (Q-TOF) instrument, equipped with a pneumatically assisted electrospray (Z-spray) ion source (Micromass, Manchester, $U K)$ operated in positive mode $(\mathrm{EV}=30 \mathrm{~V}$, $80{ }^{\circ} \mathrm{C}$, injection flow $\left.5 \mu \mathrm{L} / \mathrm{min}\right)$. NMR spectra were recorded on Bruker 600,500 or $250 \mathrm{MHz}$ spectrometer. Chemical shifts were measured in $\delta(\mathrm{ppm})$ and coupling constants $\mathrm{J}$ in $\mathrm{Hz}$ (solvent peak reference: $\delta=7.27$ for ${ }^{1} \mathrm{H}, 77.0$ for ${ }^{13} \mathrm{C}$ ). Multiplicities are indicated by s (singlet), $\mathrm{d}$ (doublet), $\mathrm{t}$ (triplet), q (quartet), $\mathrm{m}$ (multiplet) or br (broad). Chemical shifts $(\delta)$ reported are referred to internal tetramethylsilane. FT-IR spectra were recorded on Nicolet Avatar 320 FT-IR as films. Elemental analyses were performed with a Thermo Flash EA 1112 Series. 
1,2-Dideoxy-6-O-(tert-butyldiphenylsilyloxy)-3,4-di-O-isopropylidene-D-ribo-hex-1-enitol (9)

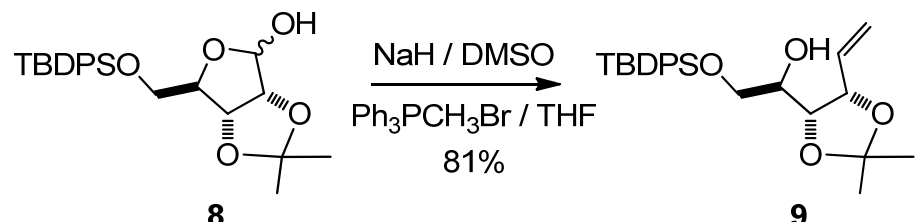

A sodium hydride suspension in mineral oil $(1.16 \mathrm{~g}, 28.9 \mathrm{mmol})$ was washed three times with $n$-hexane and then suspended in DMSO $(15.9 \mathrm{~mL})$. After heating to $68{ }^{\circ} \mathrm{C}$ for $45 \mathrm{~min}$, the grey-green mixture was transferred via syringe to a suspension of methyltriphenylphosphonium bromide (10.33 $\mathrm{g}$, $28.9 \mathrm{mmol}$, co-evaporated with toluene before use) in dry THF (110 mL). After stirring for $10 \mathrm{~min}$, a solution of lactol $8(3 \mathrm{~g}, 7.23 \mathrm{mmol})$ in dry THF $(50 \mathrm{~mL})$ was transferred via a cannula. The yellow mixture was stirred at room temperature for $16 \mathrm{~h}$ and then poured into ice water $(200 \mathrm{~mL})$. After extraction with diethyl ether $(3 \times 500 \mathrm{~mL})$, the combined organic phases were washed with a saturated $\mathrm{NaCl}$ solution $(50 \mathrm{~mL})$. The organic phase was dried with $\mathrm{MgSO}_{4}$, and after filtration and evaporation to dryness, the residue was purified by column chromatography to give $9(2.17 \mathrm{~g}, 5.1 \mathrm{mmol}, 81 \%)$ as a slightly yellow oil. $[\alpha]^{20}{ }_{\mathrm{D}}=-0.4^{\circ}\left(\mathrm{C}=1.04, \mathrm{CHCl}_{3}\right) ;{ }^{1} \mathrm{H}-\mathrm{NMR}\left(\mathrm{CDCl}_{3}\right): \delta 7.66-7.77(\mathrm{~m}, 4 \mathrm{H}, \mathrm{Ar})$, 7.34-7.51 (m, $6 \mathrm{H}, \mathrm{Ar}), 6.04$ (ddd, $\left.1 \mathrm{H}, J_{2,1 \text { trans }} 17.1 \mathrm{~Hz}, J_{2,1 \text { cis }} 10.4 \mathrm{~Hz}, J_{2,3} 6.7 \mathrm{~Hz}, \mathrm{H}-2\right), 5.44$ (d, $1 \mathrm{H}$, $J_{1 \text { trans }, 2} 17.1 \mathrm{~Hz}, \mathrm{H}-1$ trans), 5.30 (d, $\left.1 \mathrm{H}, J_{1 \mathrm{cis}, 2} 10.4 \mathrm{~Hz}, \mathrm{H}-1 \mathrm{cis}\right), 4.73$ (t, $1 \mathrm{H}, J_{3,2} 6.7 \mathrm{~Hz}, J_{3,4} 6.7 \mathrm{~Hz}$, H-3), 4.17 (dt, $\left.1 \mathrm{H}, J_{4,5} 13.7 \mathrm{~Hz}, J_{4,3} 6.7 \mathrm{~Hz}, \mathrm{H}-4\right), 3.87$ (m, $\left.2 \mathrm{H}, \mathrm{H}-6\right), 3.67-3.80$ (m, $1 \mathrm{H}, \mathrm{H}-5$ ), 2.65 (br s, $1 \mathrm{H}, \mathrm{OH}), 1.41\left(\mathrm{~s}, 3 \mathrm{H}, 1 \mathrm{CH}_{3}\right), 1.37\left(\mathrm{~s}, 3 \mathrm{H}, 1 \mathrm{CH}_{3}\right), 1.10\left(\mathrm{~s}, 9 \mathrm{H}, \mathrm{Si}-\left(\mathrm{CH}_{3}\right)_{3}\right) ;{ }^{13} \mathrm{C}-\mathrm{NMR}$ $\left(\mathrm{CDCl}_{3}\right)$ : $\delta 136.1\left(\mathrm{C}_{\text {ortho }}\right.$ or $\left.\mathrm{C}_{\text {meta }}\right), 136.0\left(\mathrm{C}_{\text {ortho }}\right.$ or $\left.\mathrm{C}_{\text {meta }}\right), 134.5(\mathrm{C}-2), 133.5\left(\mathrm{Cq}_{\mathrm{Ar}}\right), 133.5\left(\mathrm{Cq}_{\mathrm{Ar}}\right), 130.3$ $\left(\mathrm{C}_{\text {para }}\right), 128.3\left(\mathrm{C}_{\mathrm{Ar}}\right), 128.2\left(\mathrm{C}_{\mathrm{Ar}}\right), 118.1(\mathrm{C}-1), 109.2$ (Cq isopropylidene), $79.2(\mathrm{C}-3), 77.9(\mathrm{C}-4), 70.3$ (C-5), 65.7 (C-6), $28.2\left(\mathrm{CH}_{3}\right.$ isopropylidene), $27.3\left(\mathrm{C}\left(\underline{\mathrm{CH}}_{3}\right)_{3}\right), 25.9\left(\mathrm{CH}_{3}\right.$ isopropylidene $), 19.8$ $\left(\underline{\mathrm{C}}\left(\mathrm{CH}_{3}\right)_{3}\right)$; IR (neat) $v_{\max }: 3564,3481,2934,2859,1429,1218,1114,1059,704 \mathrm{~cm}^{-1}$; HRMS $(\mathrm{m} / z$, ESI) calculated for $\mathrm{C}_{25} \mathrm{H}_{34} \mathrm{O}_{4} \mathrm{NaSi}$ : $(\mathrm{M}+\mathrm{Na})=449.2124$ (calculated), 449.2120 (found).

\section{1,2-Dideoxy-3,4-di-O-isopropylidene-6-S-phenylthio-L-lyxo-hex-1-enitol (11)}

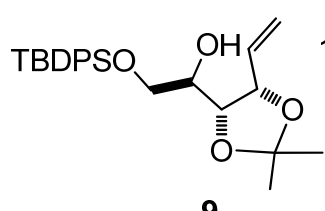

9

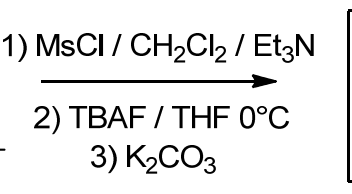

3) $\mathrm{K}_{2} \mathrm{CO}_{3}$

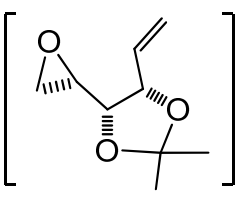

10

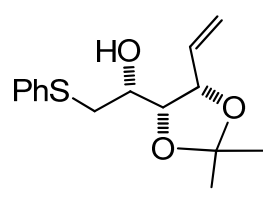

11

Triethylamine $(4.8 \mathrm{~mL}, 34.5 \mathrm{mmol})$ followed by methanesulfonyl chloride $(1.16 \mathrm{~mL}, 15 \mathrm{mmol})$ were added drop-wise to a solution of compound $9(4.89 \mathrm{~g}, 11.5 \mathrm{mmol})$ in methylene chloride $(70 \mathrm{~mL})$ at $0{ }^{\circ} \mathrm{C}$. After stirring for $16 \mathrm{~h}$ at room temperature, the mixture was diluted with diethyl ether $(300 \mathrm{~mL})$, washed with a saturated ammonium chloride solution $(50 \mathrm{~mL})$, and then with a saturated sodium chloride solution $(50 \mathrm{~mL})$. The organic phase was dried with $\mathrm{MgSO}_{4}$, filtered and evaporated to dryness. Dry THF $(110 \mathrm{~mL})$ was added and reaction mixture was cooled to $0{ }^{\circ} \mathrm{C}$. A tetra- $n$-butyl ammonium fluoride solution ( $1 \mathrm{M}$ in THF; $23 \mathrm{~mL}$ ) was added drop-wise, and the reaction mixture was stirred at room temperature for $3 \mathrm{~h}$. After addition of $\mathrm{K}_{2} \mathrm{CO}_{3}(2.8 \mathrm{~g}, 20 \mathrm{mmol})$ and stirring for $16 \mathrm{~h}$, the reaction mixture was diluted with water $(25 \mathrm{~mL})$ and extracted with diethyl ether $(3 \times 300 \mathrm{~mL})$. The combined organic phases were dried with $\mathrm{MgSO}_{4}$, filtered and evaporated to dryness. The crude 
epoxide 10 was used in the next step without further purification. Dry THF $(50 \mathrm{~mL})$ was then added and the reaction mixture was cooled to $0{ }^{\circ} \mathrm{C}$, and a THF solution of sodium thiophenoxide was added [prepared by addition of thiophenol $(2.94 \mathrm{~mL}, 28.8 \mathrm{mmol})$ to a suspension of sodium $(1.15 \mathrm{~g}$, $28.8 \mathrm{mmol})$ in dry THF $(50 \mathrm{~mL})]$. The reaction was stirred at room temperature for $16 \mathrm{~h}$. The crude mixture was then slowly quenched with methanol $(5 \mathrm{~mL})$, a half-saturated ammonium chloride solution $(30 \mathrm{~mL})$ was added and the aqueous layer was extracted with diethyl ether $(3 \times 300 \mathrm{~mL})$. The combined organic phases were dried with $\mathrm{MgSO}_{4}$, filtered and evaporated, and the residue purified by column chromatography to give $11(2.56 \mathrm{~g}, 9.1 \mathrm{mmol}, 80 \%)$ as a colorless oil. $[\alpha]^{20}{ }_{\mathrm{D}}=+27.3^{\circ}$ $\left(\mathrm{C}=1.04, \mathrm{CHCl}_{3}\right) ;{ }^{1} \mathrm{H}-\mathrm{NMR}\left(\mathrm{CDCl}_{3}\right): \delta 7.13-7.43\left(\mathrm{~m}, 5 \mathrm{H}, \mathrm{H}_{\mathrm{Ar}}\right), 5.95\left(\mathrm{ddd}, 1 \mathrm{H}, J_{2,1 \text { trans }} 17.4 \mathrm{~Hz}, J_{2,1 \text { cis }}\right.$ $\left.10.1 \mathrm{~Hz}, J_{2,3} 8.1 \mathrm{~Hz}, \mathrm{H}-2\right), 5.33$ (d, $1 \mathrm{H}, \mathrm{H}-1$ trans), 5.24 (d, $\left.1 \mathrm{H}, \mathrm{H}-1 \mathrm{cis}\right), 4.59$ (t, $1 \mathrm{H}, J_{3,4} 7.5 \mathrm{~Hz}, \mathrm{H}-3$ ), 4.27 (dd, $\left.1 \mathrm{H}, J_{4,5} 3.6 \mathrm{~Hz}, \mathrm{H}-4\right), 3.68$ (td, $\left.1 \mathrm{H}, J_{5,6} 6.5 \mathrm{~Hz}, \mathrm{H}-5\right), 3.05$ (dd, $\left.2 \mathrm{H}, \mathrm{H}-6\right), 2.44$ (br s, $1 \mathrm{H}$, $\mathrm{OH}), 1.52$ (s, $\left.3 \mathrm{H}, 1 \mathrm{CH}_{3}\right), 1.38\left(\mathrm{~s}, 3 \mathrm{H}, 1 \mathrm{CH}_{3}\right) ;{ }^{13} \mathrm{C}-\mathrm{NMR}\left(\mathrm{CDCl}_{3}\right)$ : $\delta 135.9\left(\mathrm{Cq}_{\mathrm{Ar}}\right), 134.2(\mathrm{C}-2), 130.2$ $\left(\mathrm{C}_{\text {ortho }}\right.$ or $\left.\mathrm{C}_{\text {meta }}\right), 129.4\left(\mathrm{C}_{\text {ortho }}\right.$ or $\left.\mathrm{C}_{\text {meta }}\right), 126.9\left(\mathrm{C}_{\text {para }}\right), 120.3(\mathrm{C}-1), 109.3$ (Cq isopropylidene), 79.5 (C-3 or C-4), 78.9 (C-3 or C-4), 68.8 (C-5), 38.3 (C-6), $27.5\left(\mathrm{CH}_{3}\right.$ isopropylidene), $25.3\left(\mathrm{CH}_{3}\right.$ isopropylidene); IR (neat) $v_{\text {max }}: 3480,2988,2935,1585,1482,1458,1439,1380,1215,1057,741,693 \mathrm{~cm}^{-1}$; HRMS $\left(m / z\right.$, ESI) calculated for $\mathrm{C}_{15} \mathrm{H}_{20} \mathrm{O}_{3} \mathrm{NaS}:(\mathrm{M}+\mathrm{Na})=303.1031$ (calculated), 303.1038 (found).

1,2-Dideoxy-3,4-di-O-isopropylidene-5-O-(4-methoxybenzyl)-6-S-phenylthio-L-lyxo-hex-1-enitol (12)

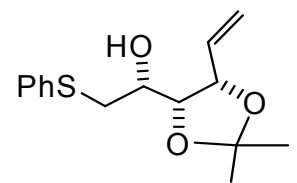

11

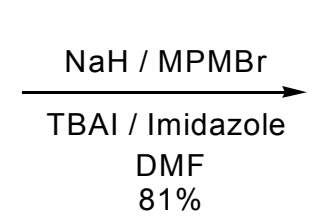

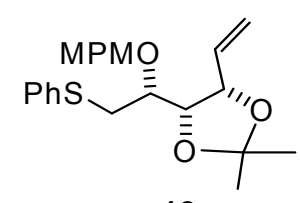

12

A solution of $11(2.38 \mathrm{~g}, 8.49 \mathrm{mmol})$ in a mixture of THF/DMF $(18 \mathrm{~mL} / 4.5 \mathrm{~mL})$ was added drop-wise to a stirring suspension of sodium hydride $(560 \mathrm{mg}, 17 \mathrm{mmol}$ ) in a mixture of THF/DMF $(18 \mathrm{~mL} / 4.5 \mathrm{~mL})$ at $0{ }^{\circ} \mathrm{C}$. After addition of tetra- $n$-butylammonium iodide $(314 \mathrm{mg}, 0.85 \mathrm{mmol})$ and imidazole (58 mg, $0.85 \mathrm{mmol}), p$-methoxybenzyl bromide $(2.48 \mathrm{~mL}, 17 \mathrm{mmol})$ was added drop-wise and the yellow suspension was stirred at room temperature for $16 \mathrm{~h}$. The reaction was quenched by the addition of methanol $(5 \mathrm{~mL})$ at $0{ }^{\circ} \mathrm{C}$ and after addition of water $(30 \mathrm{~mL})$, the reaction mixture was extracted with diethyl ether $(3 \times 300 \mathrm{~mL})$. The combined organic phases were dried with $\mathrm{MgSO}_{4}$, filtered and evaporated, and the residue purified by column chromatography to give 12 ( $2.76 \mathrm{~g}$, $6.89 \mathrm{mmol}, 81 \%)$ as a colorless oil. $[\alpha]^{20}{ }_{\mathrm{D}}=-2.8^{\circ}\left(\mathrm{C}=1.02, \mathrm{CH}_{3} \mathrm{OH}\right) ;{ }^{1} \mathrm{H}-\mathrm{NMR}\left(\mathrm{CDCl}_{3}\right): \delta 7.11-7.50$

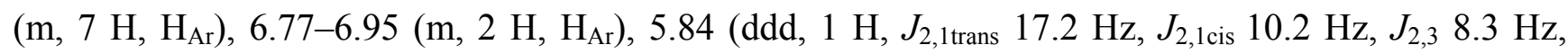
H-2), 5.26 (d, $1 \mathrm{H}, \mathrm{H}-1$ trans), 5.16 (d, $1 \mathrm{H}, \mathrm{H}-1$ cis), 4.59 (s, $2 \mathrm{H}, \mathrm{CH}_{2} \mathrm{Ph}$ ), 4.50 (dd, $1 \mathrm{H}, J_{3,4} 6.1 \mathrm{~Hz}$, H-3), 4.39 (t, $\left.1 \mathrm{H}, J_{4,5} 6.1 \mathrm{~Hz}, \mathrm{H}-4\right), 3.80$ (s, $3 \mathrm{H}, \mathrm{OMe}$ ), 3.55 (dd, $1 \mathrm{H}, J_{5,6} 11.7 \mathrm{~Hz}, \mathrm{H}-5$ ), 3.11 (dd, 2 H, $\mathrm{H}-6), 1.52\left(\mathrm{~s}, 3 \mathrm{H}, 1 \mathrm{CH}_{3}\right), 1.37\left(\mathrm{~s}, 3 \mathrm{H}, 1 \mathrm{CH}_{3}\right) ;{ }^{13} \mathrm{C}-\mathrm{NMR}\left(\mathrm{CDCl}_{3}\right): \delta 159.5\left(\mathrm{Cq}_{\mathrm{ArOMe}}\right), 136.7$ $\left(\mathrm{Cq}_{\mathrm{ArSPh}}\right), 134.8(\mathrm{C}-2), 130.9\left(\mathrm{Cq}_{\mathrm{ArCH} 2 \mathrm{O}}\right), 130.3\left(\mathrm{C}_{\mathrm{Ar}}\right), 129.8\left(\mathrm{C}_{\mathrm{Ar}}\right), 129.3\left(\mathrm{C}_{\mathrm{Ar}}\right), 126.7\left(\mathrm{C}_{\mathrm{Ar} / \mathrm{paraS}}\right), 119.6$ (C-1), 114.1 ( $\left.\mathrm{C}_{\mathrm{Ar} / \text { orthoOMe }}\right), 109.7$ (Cq isopropylidene), 80.0 (C-4), 79.5 (C-3), 77.0 (C-5), 72.8 $\left(\mathrm{CH}_{2} \mathrm{Ph}\right), 55.7(\mathrm{OMe}), 35.5(\mathrm{C}-6), 28.0\left(\mathrm{CH}_{3}\right.$ isopropylidene), $26.0\left(\mathrm{CH}_{3}\right.$ isopropylidene); IR (neat) $v_{\max }: 2986,2935,2837,1613,1614,1248,1038,742,693 \mathrm{~cm}^{-1}$; HRMS $(\mathrm{m} / z$, ESI) calculated for $\mathrm{C}_{23} \mathrm{H}_{28} \mathrm{O}_{4} \mathrm{NaS}$ : $(\mathrm{M}+\mathrm{Na})=423.1606$ (calculated), 423.1601 (found). 
3,4-Di-O-isopropylidene-2-O-(4-methoxybenzyl)-1-S-phenylthio-L-altritol (13)

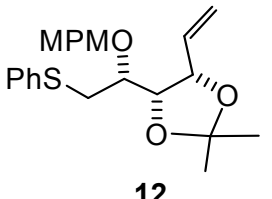

12

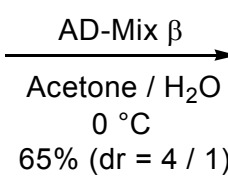

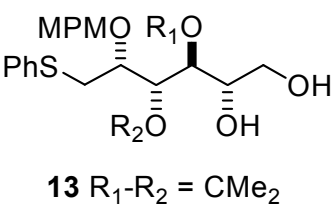

A solution of $12(654 \mathrm{mg}, 1.63 \mathrm{mmol})$ in a mixture of acetone/water (4 mL 1:1) was added to $\operatorname{AD}-$ Mix $\beta(3 \mathrm{~g})$ in a mixture of acetone/water $\left(16 \mathrm{~mL} \mathrm{1:1)}\right.$ at $0{ }^{\circ} \mathrm{C}$. After stirring for $18 \mathrm{~h}$, sodium sulfite $(3.34 \mathrm{~g})$ was added. After dilution with water $(2 \mathrm{~mL})$, the reaction was extracted with methylene chloride $(3 \times 100 \mathrm{~mL})$. The combined organic phases were dried with $\mathrm{MgSO}_{4}$, filtered and evaporated, and the residue purified by column chromatography to give 13 (368 mg, 52\%) as the major diastereoisomer, and 3,4-di- $O$-isopropylidene-2-O-(4-methoxybenzyl)-1-S-phenylthio-D-galactitol (92 mg, $13 \%$ ) as the minor one, both as colorless oils (diastereoisomeric ratio $4 / 1$ ), with $11 \%$ of recovered starting material. The same reaction with AD-Mix $\alpha$ gave $13(336 \mathrm{mg}, 47.5 \%)$ as the major diastereoisomer, and 3,4-di-O-isopropylidene-2-O-(4-methoxybenzyl)-1-S-phenylthio-D-galactitol (46 mg, 6.5\%) as the minor diastereoisomer, (diastereoisomeric ratio 7.3/1), with $20 \%$ of recovered starting material.

Major diol (13): $[\alpha]^{20}{ }_{\mathrm{D}}=-0.4^{\circ}\left(\mathrm{C}=0.975, \mathrm{CH}_{3} \mathrm{OH}\right) ;{ }^{1} \mathrm{H}-\mathrm{NMR}\left(\mathrm{CDCl}_{3}\right): \delta 7.10-7.46\left(\mathrm{~m}, 7 \mathrm{H}, \mathrm{H}_{\mathrm{Ar}}\right)$, $6.84\left(\mathrm{~d}, 2 \mathrm{H}, J_{\text {ortho,meta/OMe }} 8.6 \mathrm{~Hz}, \mathrm{H}_{\mathrm{Ar}}\right), 4.70\left(\mathrm{~d}, 1 \mathrm{H}, J_{\mathrm{CHPh}, \mathrm{CPh}} 10.9 \mathrm{~Hz}, \mathrm{CH}_{2} \mathrm{Ph}\right), 4.52(\mathrm{~d}, 1 \mathrm{H}, \mathrm{CH} 2 \mathrm{Ph})$, 4.41 (dd, 1 H, J3,4 $\left.6.1 \mathrm{~Hz}, J_{3,2} 4.3 \mathrm{~Hz}, \mathrm{H}-3\right), 4.09$ (dd, 1 H, J4,5 $\left.9.3 \mathrm{~Hz}, \mathrm{H}-4\right), 3.93-4.02$ (m, $1 \mathrm{H}, \mathrm{H}-2$ ), 3.84-3.93 (m, 1 H, H-5), 3.73-3.82 (m, 4 H, H-6a, OMe), 3.61 (dd, 1 H, J6b,6a 11.2 Hz, J6b,5 5.6 Hz, H-6b), 3.42-3.56 (br s, $1 \mathrm{H}, \mathrm{OH}), 3.46$ (dd, $\left.1 \mathrm{H}, J_{1 \mathrm{a}, 2} 5.0 \mathrm{~Hz}, J_{1 \mathrm{a}, 1 \mathrm{~b}} 13.7 \mathrm{~Hz}, \mathrm{H}-1 \mathrm{a}\right), 3.25$ (dd, $1 \mathrm{H}, J_{1 \mathrm{~b}, 2}$ $7.2 \mathrm{~Hz}, \mathrm{H}-1 \mathrm{~b}), 2.22$ (br s, $1 \mathrm{H}, \mathrm{OH}), 1.46$ (s, $3 \mathrm{H}, 1 \mathrm{CH}$ ), 1.31 (s, $\left.3 \mathrm{H}, 1 \mathrm{CH}_{3}\right) ;{ }^{13} \mathrm{C}-\mathrm{NMR}\left(\mathrm{CDCl}_{3}\right)$ : $\delta 159.9\left(\mathrm{Cq}_{\mathrm{ArOMe}}\right), 136.7\left(\mathrm{Cq}_{\mathrm{ArSPh}}\right), 130.1\left(\mathrm{Cq}_{\mathrm{ArCH} 2 \mathrm{O}}\right), 130.1\left(\mathrm{C}_{\mathrm{Ar}}\right), 129.9\left(\mathrm{C}_{\mathrm{Ar}}\right), 129.5\left(\mathrm{C}_{\mathrm{Ar}}\right), 126.7$ $\left(\mathrm{C}_{\mathrm{Ar} / \mathrm{paraS}}\right), 114.3\left(\mathrm{C}_{\mathrm{Ar} / \text { orthoOMe }}\right), 109.2$ (Cq isopropylidene), $78.0(\mathrm{C}-2), 77.8(\mathrm{C}-4), 77.6$ (C-3), 73.3 $\left(\mathrm{CH}_{2} \mathrm{Ph}\right), 70.0$ (C-5), $65.1(\mathrm{C}-6), 55.7(\mathrm{OMe}), 35.3(\mathrm{C}-1), 27.4\left(\mathrm{CH}_{3}\right.$ isopropylidene), $25.5\left(\mathrm{CH}_{3}\right.$ isopropylidene); IR (neat) $v_{\max }: 3422,2987,2933,1612,1514,1249,1070,1035,744 \mathrm{~cm}^{-1}$; HRMS $\left(m / z\right.$, ESI) calculated for $\mathrm{C}_{23} \mathrm{H}_{30} \mathrm{O}_{6} \mathrm{NaS}:(\mathrm{M}+\mathrm{Na})=457.1661$ (calculated), 457.1649 (found).

Minor diol (3,4-di-O-isopropylidene-2-O-(4-methoxybenzyl)-1-S-phenylthio-D-galactitol): ${ }^{1} \mathrm{H}-\mathrm{NMR}$ $\left(\mathrm{CDCl}_{3}\right): \delta$ 7.23-7.45 (m, $\left.5 \mathrm{H}, \mathrm{H}_{\mathrm{Ar}}\right), 7.19\left(\mathrm{~d}, 2 \mathrm{H}, J_{\text {ortho,meta/OMe }} 8.6 \mathrm{~Hz}, \mathrm{H}_{\text {ortho,meta/OMe }}\right), 6.84(\mathrm{~m}, 2 \mathrm{H}, \mathrm{H}$ meta,ortho/OMe), 4.65 (d, $\left.1 \mathrm{H}, J_{\mathrm{CHPh}, \mathrm{CPh}} 11.0 \mathrm{~Hz}, \mathrm{CH}_{2} \mathrm{Ph}\right), 4.521 \mathrm{H}$, (dd, $\left.J_{3,4} 6.6 \mathrm{~Hz}, J_{3,2} 2.0 \mathrm{~Hz}, \mathrm{H}-3\right), 4.40$ $\left(\mathrm{d}, 1 \mathrm{H}, \mathrm{CH}_{2} \mathrm{Ph}\right), 4.02$ (d, $\left.1 \mathrm{H}, \mathrm{H}-4\right), 3,80$ (s, $\left.3 \mathrm{H}, \mathrm{OMe}\right), 3.68-3.76$ (m, $1 \mathrm{H}, \mathrm{H}-2$ or H-5), 3.61-3.68 (m, $1 \mathrm{H}, \mathrm{H}-2$ or H-5), 3.56 (s, $1 \mathrm{H}, \mathrm{OH}), 3.50$ (s, $2 \mathrm{H}, \mathrm{H}-6), 3.35$ (dd, $1 \mathrm{H}, J_{1 \mathrm{a}, 2} 4.1 \mathrm{~Hz}, J_{1 \mathrm{a}, 1 \mathrm{~b}} 13.7 \mathrm{~Hz}$, H-1a), 3.22 (dd, $\left.1 \mathrm{H}, J_{1 \mathrm{a}, 2} 8.9 \mathrm{~Hz}, \mathrm{H}-1 \mathrm{~b}\right), 1.51$ (s, $3 \mathrm{H}, 1 \mathrm{CH}$ ), 1.27 (s, $3 \mathrm{H}, 1 \mathrm{CH}_{3}$ ).

6-O-(tert-Butyldimethylsilyloxy)-3,4-di-O-isopropylidene-2-O-(4-methoxybenzyl)-1-S-phenylthio-Laltritol (14)
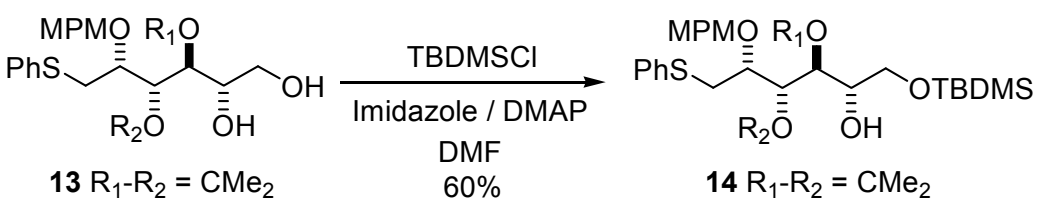
Imidazole (28 mg, $0.4 \mathrm{mmol})$, TBDMSCl (67 mg, $0.44 \mathrm{mmol})$ and DMAP (10 $\mathrm{mg}, 80 \mu \mathrm{mol})$ were added to a solution of $13(175 \mathrm{mg}, 0.4 \mathrm{mmol})$ in dry DMF $(4 \mathrm{~mL})$ at $0{ }^{\circ} \mathrm{C}$. After warming to room temperature and stirring for $6 \mathrm{~h}$, the reaction mixture was diluted with $\mathrm{CH}_{2} \mathrm{Cl}_{2}(50 \mathrm{~mL})$, washed with water $(5 \mathrm{~mL})$, and then with saturated $\mathrm{NH}_{4} \mathrm{Cl}(5 \mathrm{~mL})$. The organic phase was dried with $\mathrm{MgSO}_{4}$, filtered and evaporated, and the residue purified by column chromatography to give $\mathbf{1 4}$ (130 mg, 60\%) as a colorless oil. The crude alcohol $\mathbf{1 4}$ was used without any further purification.

6-O-(tert-Butyldimethylsilyloxy)-3,4-di-O-isopropylidene-2-O-(4-methoxybenzyl)-5-O-(R)-2-methoxyphenylacetyl-1-S-phenylthio-L-altritol (15) and 6-O-(tert-Butyldimethylsilyloxy)-3,4-di-O-isopropylidene-2-O-(4-methoxybenzyl)-5-O-(S)-2-methoxyphenylacetyl-1-S-phenylthio-L-altritol (16)
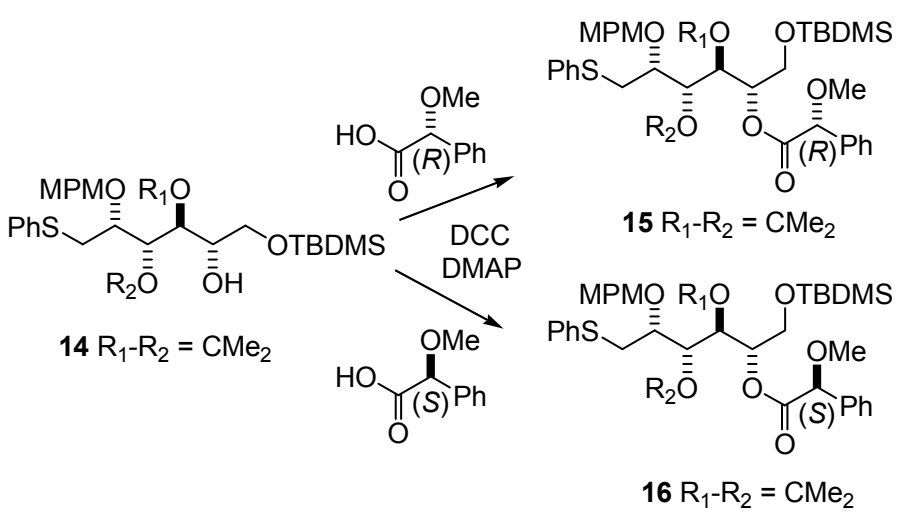

DMAP (3 mg, $20 \mu \mathrm{mol}),(-)-R$-MPA ( $80 \mathrm{mg}, 0.48 \mathrm{mmol})$ and DCC (99 $\mathrm{mg}, 0.48 \mathrm{mmol})$ were added to a solution of $14(65 \mathrm{mg}, 120 \mu \mathrm{mol})$ in dry $\mathrm{CH}_{2} \mathrm{Cl}_{2}(5 \mathrm{~mL})$ at $0{ }^{\circ} \mathrm{C}$. After stirring for $1 \mathrm{~h}$, the reaction mixture was diluted with $\mathrm{CH}_{2} \mathrm{Cl}_{2}(30 \mathrm{~mL})$, washed with saturated $\mathrm{NH}_{4} \mathrm{Cl}(5 \mathrm{~mL})$, and then with brine $(5 \mathrm{~mL})$. The organic phase was dried with $\mathrm{MgSO}_{4}$, filtered and evaporated. The residue was purified by column chromatography to give 15 as a colorless oil. ${ }^{1} \mathrm{H}-\mathrm{NMR}\left(\mathrm{CDCl}_{3}\right)$ : $\delta 7.13-7.46(\mathrm{~m}$, $\left.12 \mathrm{H}, \mathrm{H}_{\mathrm{Ar}}\right), 6.85\left(\mathrm{~d}, 2 \mathrm{H}, J_{\text {ortho,meta/OMe }} 8.6 \mathrm{~Hz}, \mathrm{H}_{\mathrm{Ar}}\right), 5.17\left(\mathrm{~m}, 1 \mathrm{H}, J_{5,4} 8.0 \mathrm{~Hz} \mathrm{H}-5\right), 4.63(\mathrm{~s}, 3 \mathrm{H}$, CHOMe), 4.58 (d, $\left.1 \mathrm{H}, J_{\mathrm{CHPh}, \mathrm{CPh}} 11.1 \mathrm{~Hz}, \mathrm{CH}_{2} \mathrm{Ph}\right), 4.50$ (d, $\left.1 \mathrm{H}, \mathrm{CH}_{2} \mathrm{Ph}\right), 4.47$ (m, $\left.2 \mathrm{H}, \mathrm{H}-3, \mathrm{H}-4\right), 3.80$ (s, 3 H, OMe), 3.73 (m, 2 H, 2 H-6), 3.44 (m, 1 H, H-2), 3.29 (m, 3 H, CHOMe), 3.23 (m, 2 H, 2 H-1), 1.46 (s, $\left.3 \mathrm{H}, 1 \mathrm{CH}_{3}\right), 1.34$ (s, $\left.3 \mathrm{H}, 1 \mathrm{CH}_{3}\right), 0.77$ (s, $\left.9 \mathrm{H}, 1 \mathrm{C}\left(\mathrm{CH}_{3}\right)_{3}\right),-0.11\left(\mathrm{~s}, 3 \mathrm{H}, \mathrm{SiCH}_{3}\right) ;-0.17$ (2s, $\left.2 \times 3 \mathrm{H}, 2 \mathrm{SiCH}_{3}\right)$. The same procedure with $(+) \mathrm{S}$-MPA gave 16 as a colorless oil. ${ }^{1} \mathrm{H}-\mathrm{NMR}\left(\mathrm{CDCl}_{3}\right)$ : $\delta 7.11-7.47\left(\mathrm{~m}, 12 \mathrm{H}, \mathrm{H}_{\mathrm{Ar}}\right), 6.82\left(\mathrm{~d}, 2 \mathrm{H}, J_{\text {ortho,meta/OMe }} 8.6 \mathrm{~Hz}, \mathrm{H}_{\mathrm{Ar}}\right), 5.20\left(\mathrm{~m}, 1 \mathrm{H}, J_{5,4} 7.2 \mathrm{~Hz} \mathrm{H}-5\right), 4.69$ (s, $3 \mathrm{H}, \mathrm{CHOMe}), 4.52$ (d, $1 \mathrm{H}, J_{\mathrm{CHPh}, \mathrm{CPh}} 11.0 \mathrm{~Hz}, \mathrm{CH}_{2} \mathrm{Ph}$ ), 4.37 (d, $1 \mathrm{H}, \mathrm{CH}_{2} \mathrm{Ph}$ ), 4.34 (dd, $1 \mathrm{H}, J_{4,3} 6.2$ Hz, H-4), 4.03 (dd, 1 H, $J_{3,2} 1.2 \mathrm{~Hz}, \mathrm{H}-3$ ), 3.91 (dd, 1 H, $\left.J_{6 \mathrm{a}, 6 \mathrm{~b}} 11.5 \mathrm{~Hz}, J_{6 \mathrm{a}, 5} 1.5 \mathrm{~Hz}, \mathrm{H}-6 \mathrm{a}\right), 3.78$ (s, $3 \mathrm{H}$, OMe), 3.76 (dd, 1 H, J6b,5 4.4 Hz, H-6b), 3.35 (m, 3 H, CHOMe), 3.12 (m, 1 H, H-2), 3.00 (dd, 1 H, $\left.J_{1 \mathrm{a}, 2} 4.5 \mathrm{~Hz}, J_{1 \mathrm{a}, 1 \mathrm{~b}} 12.7 \mathrm{~Hz}, \mathrm{H}-1 \mathrm{a}\right), 2.94$ (dd, $\left.1 \mathrm{H}, \mathrm{H}-1 \mathrm{~b}\right), 1.41$ (s, $\left.3 \mathrm{H}, 1 \mathrm{CH}_{3}\right), 1.28$ (s, $\left.3 \mathrm{H}, 1 \mathrm{CH}_{3}\right), 0.87$ $\left(\mathrm{s}, 9 \mathrm{H}, 1 \mathrm{C}\left(\mathrm{CH}_{3}\right)_{3}\right), 0.01\left(2 \mathrm{~s}, 2 \times 3 \mathrm{H}, 2 \mathrm{SiCH}_{3}\right)$.

\section{1,2-Anhydro-3,4-di-O-isopropylidene-2-O-(4-methoxybenzyl)-1-S-phenylthio-L-altritol (7)}

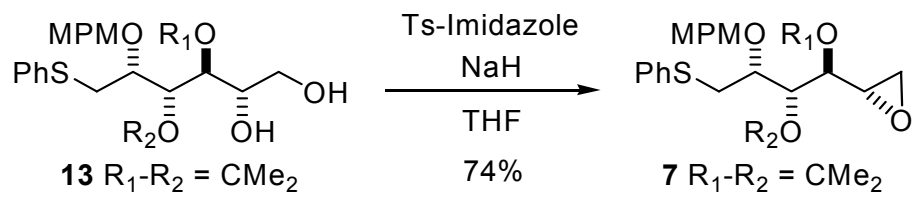


Sodium hydride 60\% suspension in mineral oil (56 mg, $1.7 \mathrm{mmol})$ was added to a solution of 13 (100 mg, $0.23 \mathrm{mmol})$ in dry THF $(2.4 \mathrm{~mL})$ at $0{ }^{\circ} \mathrm{C}$ After $15 \mathrm{~min}$ at $0{ }^{\circ} \mathrm{C}$ tosylimidazole $(104 \mathrm{mg}$, $468 \mu \mathrm{mol})$ was then added. The reaction mixture was stirred for $16 \mathrm{~h}$. After addition of diethylether $(20 \mathrm{~mL})$, washing with saturated sodium bicarbonate $(5 \mathrm{~mL})$, saturated ammonium chloride $(5 \mathrm{~mL})$ and brine $(5 \mathrm{~mL})$, the combined organic phases were dried with $\mathrm{MgSO}_{4}$, filtered and evaporated, and the residue purified by column chromatography to give $7(71 \mathrm{mg}, 74 \%)$ as a slightly yellow oil. $[\alpha]^{20}{ }_{\mathrm{D}}=-16.4^{\circ}\left(\mathrm{C}=0.22, \mathrm{CHCl}_{3}\right) ;{ }^{1} \mathrm{H}-\mathrm{NMR}\left(\mathrm{CDCl}_{3}\right): \delta 7.43\left(\mathrm{~d}, 2 \mathrm{H}, J_{\text {meta,ortho }} 7.7 \mathrm{~Hz}, \mathrm{H}_{\mathrm{Ar}}\right), 7.3(\mathrm{~m}, 4$ $\left.\mathrm{H}, \mathrm{H}_{\mathrm{Ar}}\right), 7.21\left(\mathrm{~m}, 1 \mathrm{H}, \mathrm{H}_{\mathrm{Ar}}\right), 6.87\left(\mathrm{~d}, 2 \mathrm{H}, J_{\text {ortho }} 8.5 \mathrm{~Hz}, \mathrm{H}_{\mathrm{Ar}}\right), 4.67$ (d, $\left.1 \mathrm{H}, J_{\text {gem }} 11.0 \mathrm{~Hz}, \mathrm{CH}_{2} \mathrm{Ph}\right), 4.64$ $\left(\mathrm{d}, 1 \mathrm{H}, J_{\text {gem }} 11.0 \mathrm{~Hz}, \mathrm{CH}_{2} \mathrm{Ph}\right.$ ), 4.52 (dd, $\left.1 \mathrm{H}, J_{3,4} 5.7 \mathrm{~Hz}, J_{3,2} 5.6 \mathrm{~Hz}, \mathrm{H}-3\right), 3.96$ (ddd, $1 \mathrm{H}, J_{2,1} 5.6 \mathrm{~Hz}$, H-2), 3.82 (s, $3 \mathrm{H}, \mathrm{OMe}$ ), 3.59 (dd, $\left.1 \mathrm{H}, J_{4,5} 8.1 \mathrm{~Hz}, \mathrm{H}-4\right), 3.35$ (dd, $\left.1 \mathrm{H}, J_{\text {gem }} 13.6 \mathrm{~Hz}, \mathrm{H}-1 \mathrm{a}\right), 3.25$ (dd, $1 \mathrm{H}, \mathrm{H}-1 \mathrm{~b}$ ), 3.12 (ddd, $J_{5,6 \mathrm{a}} 4.9 \mathrm{~Hz}, J_{5,6 \mathrm{~b}} 2.5 \mathrm{~Hz}, 1 \mathrm{H}, \mathrm{H}-5$ ), 2.83 (dd, 1 H, H-6a), 2.62 (dd, 1 H, H-6b), $1.53\left(\mathrm{~s}, 3 \mathrm{H}, 1 \mathrm{CH}_{3}\right), 1.36\left(\mathrm{~s}, 3 \mathrm{H}, 1 \mathrm{CH}_{3}\right) ;{ }^{13} \mathrm{C}-\mathrm{NMR}\left(\mathrm{CDCl}_{3}\right): \delta 159.2\left(\mathrm{Cq}_{\mathrm{ArOMe}}\right), 136.3\left(\mathrm{Cq}_{\mathrm{ArSPh}}\right)$, $130.4\left(\mathrm{Cq}_{\mathrm{ArCH} 2 \mathrm{O}}\right), 129.6\left(2 \mathrm{C}_{\mathrm{Ar}}\right), 128.9\left(\mathrm{C}_{\mathrm{Ar}}\right), 126.2\left(\mathrm{C}_{\mathrm{Ar} / \mathrm{paraS}}\right), 113.7\left(\mathrm{C}_{\mathrm{Ar} / \mathrm{orthoOMe}}\right), 109.2(\mathrm{Cq}$ isopropylidene), $78.8(\mathrm{C}-4), 78.7(\mathrm{C}-3), 76.3(\mathrm{C}-2), 72.3\left(\mathrm{CH}_{2} \mathrm{Ph}\right)$, s55.3 $(\mathrm{OMe}), 49.4(\mathrm{C}-5), 46.1$ (C-6), 35.1 (C-1), $27.6\left(\mathrm{CH}_{3}\right.$ isopropylidene), $25.3\left(\mathrm{CH}_{3}\right.$ isopropylidene); IR (neat) $v_{\max }$ : 3057, 2989, 2933, 1653, 1615, 1559, 1540, 1515, 1248, 1086, 1038, $741 \mathrm{~cm}^{-1}$; HRMS (m/z, ESI) calculated for $\mathrm{C}_{23} \mathrm{H}_{28} \mathrm{O}_{5} \mathrm{NaS}:(\mathrm{M}+\mathrm{Na})=439.1555$ (calculated), 439.1557 (found).

\section{Conclusions}

We have developed a short and efficient procedure for the synthesis of a diversely protected rare L-altritol derivative 7 (which could also be seen as a L-talitol derivative). This key compound offers access to the central stereoclusters of the sagittamide backbones. Work is in progress to extend this efficient pathway to making useful fragments in order to definitively establish the exact configurations of these natural substances.

\section{Acknowledgments}

A. Humbert would especially like to thank the Ministry of Higher Education and Research (MESR) for her $\mathrm{PhD}$ scholarship. Financial support from the CNRS, the Conseil Regional Champagne Ardenne, the Conseil General de la Marne, and the EU-program FEDER for the PlAneT CPER project is also gratefully acknowledged.

\section{References}

1. Lievens, S.C.; Molinski, T.F. Sagittamides A and B. Polyacetoxy Long-Chain Acyl Amino Acids from a Didemnid Ascidian. Org. Lett. 2005, 7, 2281-2284.

2. Lievens, S.C.; Morinaka, B.I.; Molinski, T.F. Stereochemical Elucidation of New Sagittamides C-F from a Didemnid Ascidian. Aust. J. Chem. 2010, 63, 935-941.

3. Seike, H.; Ghosh, I.; Kishi, Y. Stereochemistry of Sagittamide A: Prediction and Confirmation. Org. Lett. 2006, 8, 3865-3868.

4. Lievens, S.C.; Molinski, T.F. Stereochemical Elucidation of New Sagittamides C-F from a Didemnid Ascidian. J. Am. Chem. Soc. 2006, 128, 11764-11765. 
5. Schuetz, A.; Junker, J.; Leonov, A.; Lange, O.F.; Molinski, T.F.; Griesinger, C. Stereochemical Elucidation of New Sagittamides C-F from a Didemnid Ascidian. J. Am. Chem. Soc. 2007, 129, 15114-15115.

6. Nuzillard, J.-M.; Banchet, A.; Haudrechy, A. Application of the Quiral Program to the Challenge of Myoinositol Synthesis. J. Chem. Inf. Model. 2007, 47, 1979-1985.

7. Nuzillard, J.-M.; Haudrechy, A. Quiral: A computer program for the synthesis of chiral molecules from sugars. Tetrahedron Lett. 2007, 48, 2311-2313.

8. Stewart, A.O.; Williams, R.M. C-Glycosidation of pyridyl thioglycosides. J. Am. Chem. Soc. 1985, 107, 4289-4296.

9. Choi, W.J.; Moon, H.R.; Kim, H.O.; Yoo, B.N.; Lee, J.A.; Shin, D.H.; Jeong, L.S. Preparative and Stereoselective Synthesis of the Versatile Intermediate for Carbocyclic Nucleosides: Effects of the Bulky Protecting Groups to Enforce Facial Selectivity. J. Org. Chem. 2004, 69, 2634-2636.

10. Swamy, K.C.K.; Kumar, N.N.B.; Balaraman, E.; Kumar, K.V.P.P. Mitsunobu and Related Reactions: Advances and Applications. Chem. Rev. 2009, 109, 2551-2651.

11. Dodge, J.A.; Trujillo, J.I.; Presnell, M. Effect of the acidic component on the Mitsunobu inversion of a sterically hindered alcohol. J. Org. Chem. 1994, 59, 234-236.

12. Saïah, M.; Bessodes, M.; Antonakis, K. The use of chloroacetic acid in the mitsunobu reaction. Tetrahedron Lett. 1992, 33, 4317-4320.

13. Guillarme, S.; Ple, K.; Haudrechy, A. Selective Synthesis of $\alpha$-C-(Alkynyl)-galactosides by an Efficient Tandem Reaction. J. Org. Chem. 2006, 71, 1015-1017.

14. Banchet, A.; Guillarme, S.; Haudrechy, A. Mitsunobu and Related Reactions: Advances and Applications. Synlett 2007, 9, 1467-1469.

15. Martinez, A.; Hénon, E.; Coiffier, C.; Banchet, A.; Harakat, D.; Nuzillard, J.-M.; Haudrechy, A. An unexpected rearrangement giving a new thiosubstituted carbohydrate. Carbohydr. Res. 2010, 345, 1088-1093.

16. Jang, K.P.; Choi, S.Y.; Chung, Y.K.; Lee, E. Synthetic Studies on Lytophilippine A: Synthesis of the Proposed Structure. Org. Lett. 2011, 13, 2476-2479.

17. Kartika, R.; Gruffi, T.R.; Taylor, R.E. Concise Enantioselective Total Synthesis of Neopeltolide Macrolactone Highlighted by Ether Transfer. Org. Lett. 2008, 10, 5047-5050.

18. Walsh, P.J.; Tong Ho, P.; King, S.B.; Sharpless, K.B. Asymmetric dihydroxylation of olefins containing sulfur: Chemoselective oxidation of $\mathrm{C}=\mathrm{C}$ double bonds in the presence of sulfides, 1,3-dithianes, and disulfides. Tetrahedron Lett. 1994, 35, 5129-5132.

19. Sammakia, T.; Hurley, T.B.; Sammond, D.M.; Smith, R.S.; Sobolov, S.B.; Oeschger, T.R. Dihydroxylation and oxidative cleavage of olefins in the presence of sulfur. Tetrahedron Lett. 1996, 37, 4427-4430.

20. Dong, S.; Paquette, L.A. Stereoselective Synthesis of Conformationally Constrained 2'-Deoxy-4'thia $\beta$-Anomeric Spirocyclic Nucleosides Featuring Either Hydroxyl Configuration at C5'. J. Org. Chem. 2005, 70, 1580-1596.

21. Wu, M.-J.; Lee, C.-L.; Wu, Y.-C.; Chen, C.-P. Synthesis of $(4 R, 15 R, 16 R, 21 S)$-rollicosin and its 4S epimer. Eur. J. Org. Chem. 2008, 5, 854-861. 
22. Sharma, P.K.; He, M.; Jurayj, J.; Gou, D.-M.; Lombardy, R.; Romanczy, L.J.; Schroeter, H. Enantioselective Syntheses of Sulfur Analogues of Flavan-3-Ols. Molecules 2010, 15, 5595-5619.

23. Iwashima, M.; Kinsho, T.; Smith Iii, A.B. A caveat on the Sharpless asymmetric dihydroxylation. Tetrahedron Lett. 1995, 36, 2199-2202.

24. Seco, J.M.; Quiñoá, E.; Riguera, R. An unexpected rearrangement giving a new thiosubstituted carbohydrate. Tetrahedron: Asymmetry 2001, 12, 2915-2925.

Sample Availability: Contact the authors.

(C) 2012 by the authors; licensee MDPI, Basel, Switzerland. This article is an open access article distributed under the terms and conditions of the Creative Commons Attribution license (http://creativecommons.org/licenses/by/3.0/). 